\title{
Search for Gamma-ray Counterparts of Newly Discovered Radio Astrophysical Sources
}

\author{
S. Best, J. L. Bazo Alba* \\ Sección Física, Departamento de Ciencias, Pontificia Universidad Católica del Perú, Av. \\ Universitaria 1801, Lima 32, Perú \\ E-mail: jbazo@pucp.edu.pe
}

\begin{abstract}
We study two newly discovered classes of radio sources: the highly energetic, short-lived events, known as Fast Radio Bursts (FRBs), and a new category of compact radio galaxies known as Fanaroff-Riley type 0 (FR0s). Due to the unknown nature of FRBs and a previous correlation found with an FR0 in $\gamma$-rays, it is possible that these sources could also emit high energy photons in the Fermi-LAT energy range. Here we present an exhaustive time-dependent and spatial search of all up-to-date observed FRBs and FR0s, respectively. We perform a likelihood analysis of the radio sources by modeling the excess flux of gamma rays with a power law using data from Fermi-LAT and the 4FGL catalog. Sources with a pre-trial significance greater than $4 \sigma$ were further analyzed including 2 FRBs and 7 FR0s. No correlations with more than $5 \sigma$ were found after taking into account nearby sources and trial corrections. Therefore, upper limits for all sources were calculated.
\end{abstract}

36th International Cosmic Ray Conference -ICRC2019-

July 24th - August 1st, 2019

Madison, WI, U.S.A.

${ }^{*}$ Speaker. 


\section{Introduction}

Multi-wavelength searches using different satellites and ground-based telescopes open new windows of opportunity for astronomy. The possibility to observe the same source through different parts of the electromagnetic spectrum can give us valuable information about their origin and processes occurring inside them. The sources we study: Fast Radio Bursts (FRBs) and FanaroffRiley type 0 galaxies (FR0s) were first discovered in radio. However, it is possible that these radio sources could also emit in a broader spectrum reaching up to gamma rays in the Fermi-LAT energy range (20 MeV - $300 \mathrm{GeV})$.

We search for such gamma-ray counterpart using a binned and unbinned likelihood analysis with Fermi-LAT data. We find for each source the optimal set of parameters which maximizes the Test Statistic (TS) using a power law model for the possible gamma-ray flux. Detailed information and further analysis of the sources with the highest TS $(\geq 16)$ are also presented.

\section{Radio Sources}

Fast Radio Bursts are very energetic, millisecond radio intense events which are considered extragalactic due to their spatial distribution and dispersion measure (DM) [1]. Only one FRB (FRB121102), a repeating burst source, has been correlated with a known small dwarf galaxy [2]. These sources could emit gamma-rays as well. Possible origins for these gamma-ray emission signals are gamma-ray bursts or neutron star merging [3] which could also be observed in gravitational waves. Since these emissions could occur before or after the radio signal we look for correlations around the arrival time of the radio emission. Some FRBs were found during searches for other astronomical events, such as the Parkes Telescope search for pulsars, which could make them correlate (e.g: FRB180309, FRB180311) [4].

The first FRB was discovered in 2007 by Lorimer [1], but only recently new telescopes have increased the number of detected FRBs. For telescopes like CHIME the rate is predicted to be between 2 to 50 FRBs per day.

Fanaroff-Riley type 0 radio galaxies is a new category added in 2015 [5] to the original Fanaroff-Riley classification. This new category contains an increasing number of sources which did not match well with the FRI class. Fanaroff-Riley compact radio galaxies are divided into type I (low intensity, with the bulk of the radio emissions coming from the core) and type II (high intensity with luminosity increasing in the lobes). FR0 galaxies are similar to type I except for a large deficit of extended radio structures [6].

We analize a subset of 108 FR0 sources catalogued [6] as part of the SSDS survey and 76 FRB events which have been detected by several telescopes (ASKAP, Parkes and UTMOST in Australia and CHIME in Canada) [8]. The mean free path for these energies is within the expected distance for these sources [9].

There have been previous FBR searches in other wavelengths such as X-rays [10] and $\gamma$-rays ([11], [12]). One correlation of an FR0 with gamma rays of a Fermi source, 3FGLJ1330.0-3818, has already been found [7]. However, our analysis (temporal in the case of FRBs and spatial in the case of FR0s) is more exhaustive, since it is applied to all sources listed in the available catalogs mentioned above. 


\section{Fermi-LAT data analysis}

The LAT, Large Area Telescope, on board of the Fermi space observatory, is an imaging gamma-ray detector that uses pair production to measure energy and momentum of high energy incident photons ( $20 \mathrm{MeV}-300 \mathrm{GeV}$ ). It covers, at any given moment, around $20 \%$ of the sky with a resolution of arcminutes and has been collecting data since 2008 [13].

We used Fermi LAT Pass 8 data with Fermi Science Tools cf201901. We extracted photon data from $100 \mathrm{MeV}$ to $300 \mathrm{GeV}$ and spacecraft history using the time and coordinates provided by the FRB [8] and FR0 [6] catalogs with an Astroquery [14] script. For FRBs we extracted data 25 hours around the radio event. For FR0s we took one year of recent data (03-18 to 03-19).

To reduce contamination from Earth's limb emission we used a maximum zenith angle $\left(90^{\circ}\right.$ for FR0s and $100^{\circ}$ for FRBs to increase the photon count) and took events only when the spacecraft was operating correctly ( $[$ DAT \&\& QUALITY] $==1$ ). We then selected for events corresponding to all SOURCE class events (evclass $=128$ and evtype $=3$ ) and used the IRFs (Instrument Response Function): P8R3_SOURCE_V2. We took a ROI (region of interest) of $15^{\circ}$ and set the background sources using the 4FGL catalog [15]. For convergence purposes, all source's index were fixed to their catalog value and only normalization was left free to vary up to $10^{\circ}$ from the center, after which normalizations were fixed. We used gll_iem_v06 to model the galactic background and iso_P8R3_SOURCE_V2_v1 for the extragalactic background.

For both sources we modeled the filtered data using a Power Law (Eq. 3.1) with a varying $\Gamma$ index from 1.75 to 3.0 in steps of 0.25 , with a fixed energy scale $E_{0}$ of $100 \mathrm{MeV}$.

$$
\frac{d N}{d E}=N_{0}\left(\frac{E}{E_{0}}\right)^{-\Gamma}
$$

We used a time-dependent search for FRBs. Due to the low photon count, an unbinned likelihood analysis was applied to each one hour bin, in which the photon data was divided. A spatial search, varying the source location by $0.1^{\circ}$ in a grid of $1.1^{\circ} \times 1.1^{\circ}$, was applied to the FR0s. In this case we used a binned likelihood analysis due to the large photon count for each source.

For each case we get a TS (i.e. likelihood-ratio test) defined by:

$$
T S=-2 \log \left(\frac{L \mid \theta_{0}}{L \mid \theta_{M L}}\right)
$$

where $L$ represents the likelihood given $\theta_{0}$, the set of parameters representing the null hypothesis, and $\theta_{M L}$, the most likely value of the set of parameter given the data. The square root of the TS can be treated as the detection significance, since we are fitting one parameter (i.e. $\theta_{0}=N_{0}$ ).

More tests were performed for the sources with the highest TS $(\geq 16)$. We applied an exponential cutoff model, given in Eq. 3.3, extended the TS spatial map for FR0s, and analyzed their light curve and spectral energy distributions. In the case of the exponential cutoff model, we looped over both the additional $\Gamma_{2}$ indices, the energy cutoff, $E_{C}$, and left the normalization, $N_{0}$, to vary freely. $\Gamma$ was fixed to the value which gave the highest TS in the power law analysis.

$$
\frac{d N}{d E}=N_{0}\left(\frac{E}{E_{0}}\right)^{-\Gamma} \exp \left(-\left(\frac{E}{E_{C}}\right)^{-\Gamma_{2}}\right)
$$


Table 1. Parameters for the maximum TS of the most significant $(>4 \sigma)$ FRBs and FR0s.

\begin{tabular}{cccccccc}
\hline \hline Source Name & $\begin{array}{c}\text { Index } \\
(\Gamma)\end{array}$ & $\begin{array}{c}\text { Time } \\
(\mathrm{h})\end{array}$ & $\begin{array}{c}\text { R.A. A. } \\
\left({ }^{\circ}\right)\end{array}$ & $\begin{array}{c}\text { Dec } \\
\left({ }^{\circ}\right)\end{array}$ & $\begin{array}{c}\text { Flux }\left(10^{-9}\right) \\
\left(\mathrm{ph} \mathrm{cm}^{-2} \mathrm{~s}^{-1}\right)\end{array}$ & TS & $\begin{array}{c}\text { p-value } \\
\text { post-trial }\end{array}$ \\
\hline FRB150807 & 3.0 & 8.0 & & & $1.92 \times 10^{3}$ & 17.84 & $1.8 \times 10^{-3}$ \\
FRB171004 & 3.0 & -5.0 & & & $2.30 \times 10^{3}$ & 19.82 & $6.5 \times 10^{-4}$ \\
SDSS_J093003.56+341325.3 & 3.0 & & 0.5 & 0.5 & 18.40 & 18.60 & $1.7 \times 10^{-3}$ \\
SDSS_J103719.33+433515.3 & 3.0 & & -0.4 & 0.5 & 18.82 & 26.61 & $3 \times 10^{-5}$ \\
SDSS_J104811.90+045954.8 & 3.0 & -0.5 & 0.5 & 23.84 & 21.20 & $4.3 \times 10^{-4}$ \\
SDSS_J135226.71+140528.5 & 2.0 & & 0.3 & 0.5 & 3.74 & 19.01 & $1.4 \times 10^{-3}$ \\
SDSS_J150601.89+084723.2 & 3.0 & & -0.5 & 0.5 & 31.81 & 25.24 & $5 \times 10^{-5}$ \\
SDSS_J150636.57+092618.3 & 3.0 & & -0.5 & 0.5 & 55.95 & 60.47 & $<10^{-10}$ \\
SDSS_J155951.61+255626.3 & 3.0 & & -0.2 & -0.5 & 19.37 & 24.00 & $1 \times 10^{-4}$ \\
\hline
\end{tabular}

Note. - Column description: (1) Source name; (2) $\Gamma$ index of the power law; (3) Time difference between FRB radio event and the bin central time; (4) (5) Distance to the right ascension (declination) component of the source's position used in the fitting model of the FR0; (6) Flux upper limit (95\% confidence level); (7) Maximum TS found for the source; (8) p-value after trial corrections.

\section{Results}

The results of our search for the most significant sources (TS $\geq 16$ which corresponds to roughly $4 \sigma$ ) can be found summarized in Table 1 . For the complete results see [16]. There are only 2/76 FRBs and 7/108 FR0s satisfying this criteria. We present for each source the set of parameters which maximizes the TS, including the $\Gamma$ index of the fitted power law (see Eq. 3.1). In addition, in the case of FRBs, the time from the radio event is given, corresponding to the highest TS. For FR0s the distance from the detected radio source, in right ascension and declination, is shown. The resulting maximum TS is also presented as sky maps for all sources in galactic coordinates in Fig. 1 for FRBs and FR0s. No source is more significant than $5 \sigma$ after trial corrections for the numbers of sources searched, but for one SDSS_J150636.57+092618.3, which is later identified with a strong nearby source.

We did further tests on the promising sources listed in Table 1. In Fig. 2 we show the light curves with flux upper limits of the FRBs and their corresponding TS in color scale. No significant enhancement (i.e. flare) around the time of the event is observed. In addition, we analyzed the source's SED (spectral energy distribution) for both FRBs. FRB150807 had data only for one energy bin and FRB171004 contributed for low energies (few hundred MeV) at the level of a nearby source to the total model.

We also tested an exponential cutoff model for both FRBs using (Eq. 3.3) with $\Gamma_{2}$ index (from 1.0 to 2.5 in 0.5 steps), energy cutoff, $E_{C}$ (from $10^{3}$ to $10^{6}$ in 5 logarithmically spaced intervals), and fitted the normalization, $N_{0}$. $\Gamma$ was fixed to the value which gave the highest TS in the power law analysis. For both FRBs the results were less significant than with the simpler model. 


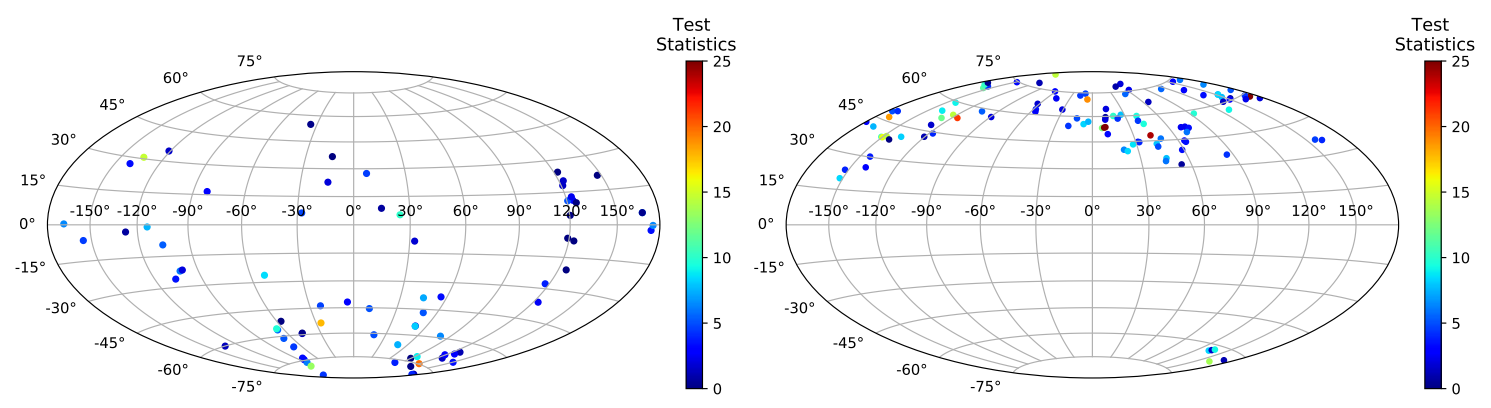

Figure 1 Sky map in galactic coordinates of the maximum TS found according to the color scale for: (upper) 76 FRBs, where the TS corresponds to the best combination of time from the FRB event and $\Gamma$ index of the power law and (lower) 108 FR0s, where the TS corresponds to the best combination of the source's position in a spatial grid and $\Gamma$ index of the power law.
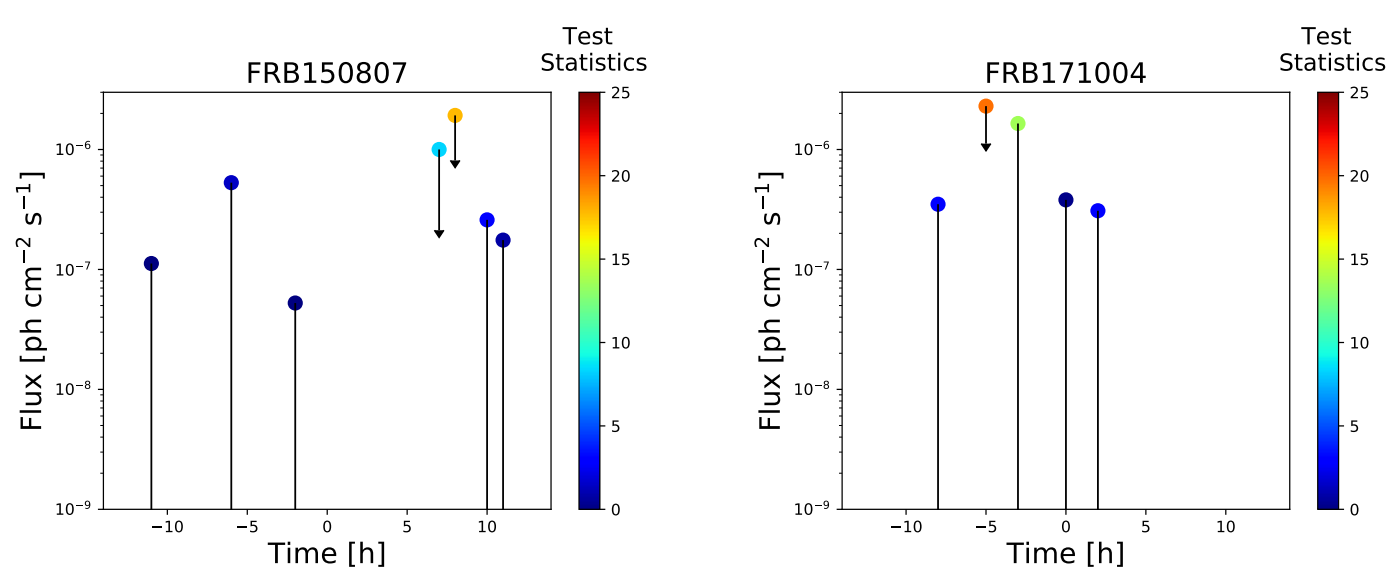

Figure 2 Light curves of the two most significant FRBs showing flux upper limits. The time axis is centered at the radio event's detection time using a 1 hour bin. The $\Gamma$ index corresponds to highest TS found in one of the time bins and the color represents the TS. The points that do not appear are due to the fit not converging. Left: FRB150807, max. TS $=17.84(\Gamma=3.0,8$ hours after the radio event). Right: FRB171004, max. TS $=19.82(\Gamma=3.0,5$ hours before the radio event).

Then, we analyzed the SEDs of the seven FR0s showing examples in Fig. 3. The counts for these sources are much lower compared to nearby sources and the SED is actually dominated by the galactic and extragalactic backgrounds. This characteristic is shared by all 7 FR0s.

We compared the areas of high TS with known nearby sources from the 4FGL catalog. We found a possible origin for 4 FR0s, shown in Table 2. For the remaining 3 sources we checked the count map and did not find a possible origin.

In addition, we extended the TS map in the direction of the suspected high TS origin. The source position was varied from pixel to pixel by $0.1^{\circ}$ in a $1.5^{\circ} \times 1.5^{\circ}$ grid, where the $\left(0^{\circ}, 0^{\circ}\right)$ position is the FR0 catalog location. In Fig. 4 the apparent sources continue as expected for SDSS_J104811.90+045954.8 and SDSS_J155951.61+255626.3. For SDSS_J135226.71+140528.5. However, the high TS region does not continue in the extended map (only two isolated points show high TS) which could be attributed to a statistical fluctuation and it is thus not shown here. 
Table 2. Possible origin to the high TS for FR0s

\begin{tabular}{ccccc}
\hline \hline Source Name & Nearby source & $\begin{array}{c}\text { R.A. } \\
\text { distance }\left(^{\circ}\right)\end{array}$ & $\begin{array}{c}\text { Dec. } \\
\text { distance }\left({ }^{\circ}\right)\end{array}$ & $\begin{array}{c}\text { Total } \\
\text { distance }\left(^{\circ}\right)\end{array}$ \\
\hline SDSS_J093003.56+341325.3 & 4FGL J0930.7+3502 & 0.167 & 0.610 & 0.821 \\
SDSS_J103719.33+433515.3 & 4FGL J1035.6+4409 & 0.907 & 0.073 & 0.644 \\
SDSS_J150601.89+084723.2 & 4FGL J1504.4+1029 & 0.904 & 1.208 & 1.754 \\
SDSS_J150636.57+092618.3 & 4FGL J1504.4+1029 & 1.049 & 0.559 & 1.189 \\
\hline
\end{tabular}
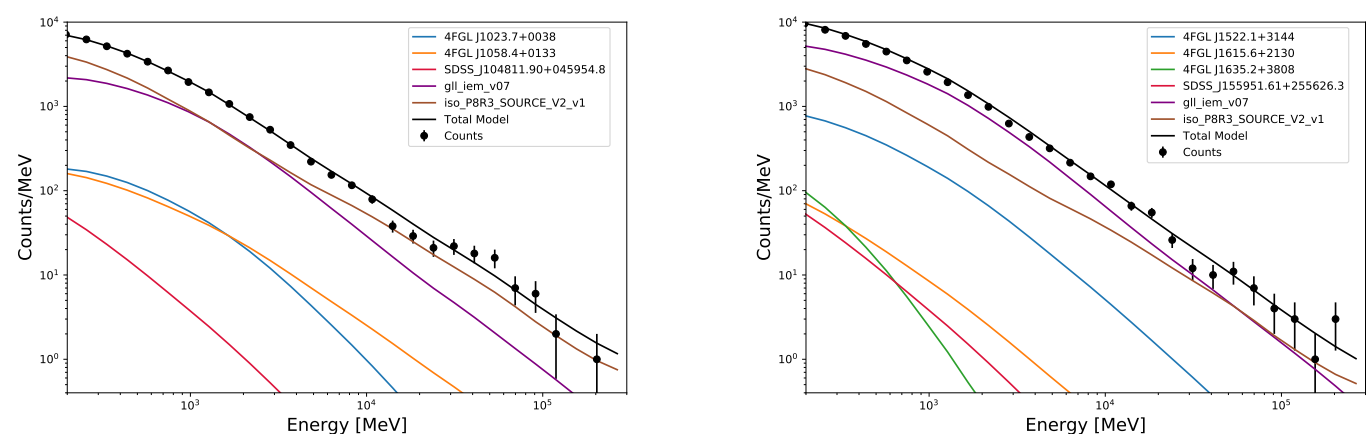

Figure 3 Spectral energy distribution of SDSS_J104811.90+045954.8 (left) and SDSS_J155951.61+255626.3 (right) using $\Gamma=3$ and position corresponding to the maximum TS. Points are Fermi data. The red line is the total model (i.e. the sum of the individual fits), representing the studied sources. The other lines correspond to the most contributing sources.
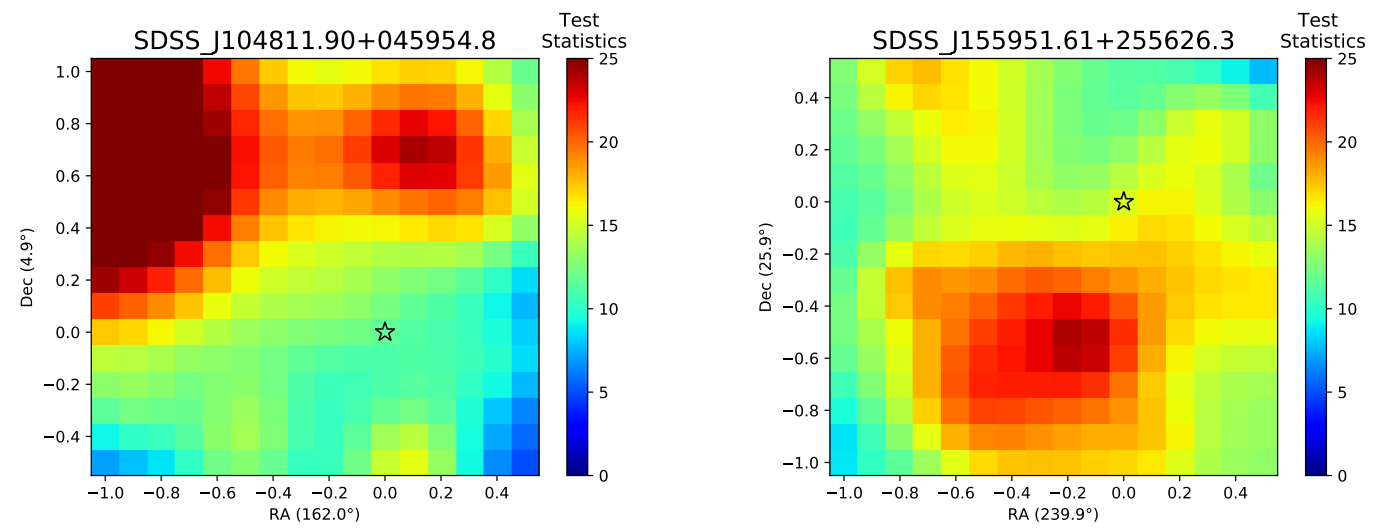

Figure 4 Test statistics extended spatial grid maps of Left: SDSS_J104811.90+045954.8, Right: SDSS_J155951.61+255626.3. Each pixel in the map represents a displacement of $0.1^{\circ}$ from the detected central position of the source, marked with a star, in right ascension and declination. The results correspond to the $\Gamma$ index that gave the maximum TS(Left: 3.0, Right: 3.0). 


\section{Conclusions}

We analyzed 76 FRBs and 108 FR0s, extragalactic sources discovered in radio, searching for a correlation with Fermi-LAT gamma ray data. We performed time-dependent (FRB) and spatial (FR0) searches using an unbinned and binned likelihood analyses fixing the $\Gamma$ index of a power law that describes their flux, only letting one parameter (i.e. normalization) vary.

From these, 9 sources (two FRBs and seven FR0s) had a TS $>16$ (roughly $4 \sigma$ pre-trial). FRB150807 has only data in one energy bin of its SED and FRB171004 has a post-trial significance of less than $4 \sigma$. From the seven FR0s, 4 have nearby sources that could explain their high TS. From the remaining 3, SDSS_J135226.71+140528.5 has high TS in only two isolated points of the grid suggesting a statistical fluctuation. The remaining two FR0s (SDSS_J104811.90+045954.8 and SDSS_J155951.61+255626.3) could be discarded due to their SED, since in both cases the background is dominant. The absence of a $\gamma$-ray signal from FR0s is in agreement with expectations that these objects will be too faint in gamma-rays to be detected individually [17]. Therefore, upper limits for all sources with $95 \%$ confidence level have been calculated.

\section{Acknowledgments}

S.B. acknowledges support from CONCYTEC-FONDECyT scholarship under grant 233-20152. J.B. acknowledges funding by the DGI at PUCP under grant 2017-3-0019.

\section{References}

[1] Lorimer, D. R., Bailes, M., McLaughlin, M. A., Narkevic, D. J., \& Crawford, F. 2007, Sci, 318, 5851

[2] Chatterjee, S., Law, C. J., Wharton, R. S., et al. 2017, Nature, 337, 541, 58-61

[3] Dokuchaev, V. I., Eroshenko, Y. N. 2017, Int. J. Mod. Phys. A, 2, 11-21

[4] Oslowski, S. 2018, http://www.astronomerstelegram.org/?read=11396

[5] Baldi, R. D., Capetti, A., \& Giovannini, G. 2015, AN, 337, Issue 1-2

[6] Baldi, R. D., Capetti, A., \& Massaro, F. 2017, A\&A 609, A1

[7] Grandi, P., Capetti, A., Baldi, R. D. 2015, MNRAS, 457, 1

[8] Petroff, E., Barr, E. D., Jameson, A., et al. 2016, Publ. Astron. Soc. Aust., 33, e045, frbcat .org

[9] Roncadelli, M., de Angelis, A., Galanti, G. 2012, Journal of Physics: Conference Series, 375

[10] Scholz, P., Bogdanov, S., Hessels, J. W., et al. 2017, ApJ, 846, 1

[11] Xi, S. Q., Tam, P. H. T., Peng, F. K., Wang, X. Y. 2017, ApJL, 842, 1

[12] Yamasaki, S., Totani, T., Kawanaka, N. 2016, MNRAS, 460, 3

[13] Atwood, W. B., Abdo, A. A., Ackermann, M., et al. 2009, ApJ, 697, 1071

[14] Ginsburg, A., Astroquery 2019, https: //astroquery.readthedocs.io/en/latest/

[15] Fermi-LAT Collab. 2019, arXiv:1902.10045

[16] Best, S., Bazo, J. 2019, arXiv:1906.01664

[17] Stecker, F., Shrader, C., Malkan, M. 2019, arXiv:1903.06544 